\title{
Chemical Trends of Schottky Barrier Behavior on monolayer hexagonal B, Al and Ga Nitrides
}

\author{
Haichang $\mathrm{Lu}^{1}$, Yuzheng Guo ${ }^{2}$, John Robertson ${ }^{1 *}$ \\ ${ }^{1}$ Department of Engineering, Cambridge University, Cambridge CB2 1PZ, United Kingdom \\ ${ }^{2}$ Rowland Institute, Harvard University, Cambridge, MA, 02142, United States
}

KEYWORDS: Hexagonal boron nitride, metal contact, Schottky barrier, 2D semiconductor, density functional theory

\begin{abstract}
The Schottky Barrier Heights (SBH) of metal layers on top of monolayer hexagonal Xnitrides $(\mathrm{X}=\mathrm{B}, \mathrm{Al}, \mathrm{Ga}, \mathrm{h}-\mathrm{XN})$ are calculated using supercells and density functional theory so as to understand the chemical trends of contact formation on graphene and the 2D layered semiconductors such as the transition metal dichalcogenides. The Fermi level pinning factor $\mathrm{S}$ of SBHs on h-BN is calculated to be near 1, indicating no pinning. For h-AlN and h-GaN, the calculated pinning factor is about 0.63 , less than for $\mathrm{h}-\mathrm{BN}$. We attribute this to the formation of stronger, chemisorptive bonds between the nitrides and the contact metal layer. Generally the h-BN layer remains in a planar $\mathrm{sp}^{2}$ geometry and has weak physisorptive bonds to the metals, whereas h-AlN and h-GaN buckle out of their planar geometry which enables them to form the chemisorbtive bonds to the metals.
\end{abstract}

\section{INTRODUCTION}

Two-dimensional (2D) layered semiconductors have been proposed for use in future electronic devices including tunnel field effect transistors (FETs) and low power sensors [13]. However, their device performances tend to be limited by contact resistances, due to the presence of Schottky barriers at their contacts [4,5]. In some cases such as $\mathrm{MoS}_{2}$, the Schottky barrier heights (SBHs) show strong Fermi level pinning (FLP) [5-11], which limits our ability to choose a contact metal with a suitable work function so as to reduce the SBH. In other cases like h-BN, there is little FLP according to theoretical calculations [12]. The behavior of SBHs in two-dimension systems is often attributed to their dimensionality and to the presence of van der Waals interlayer bonding [13-15]. However, it is interesting to understand how much of the behavior depends on dimensionality, and how much depends on the chemical bonding between their layers. We therefore study the chemical trends in the system of the 2-dimensional (2D) hexagonal group III-nitrides, h-BN, h-AlN and h-GaN, which show both types of behavior.

In general, FLP can arise from either intrinsic or extrinsic effects $[9,16]$. The extrinsic effects are due to atomic defects such as vacancies created by the formation of the contacts, which in principle can be avoided and will not be considered further here. The intrinsic mechanism is due charge transfer between the metal and the nitride, via the travelling wave states of the metal extending into the semiconductor band gap, where they are called 'metal induced gap states' or MIGS [16-19]. The MIGS can pin the Fermi level if their density is large enough, and if they have not decayed too much across any bonding 'gap' between the contacts and the semiconductor layer [6,13]. Thus, the question turns out to be what is the actual bonding between the contact metal and 2D layer in each case, and how it varies in the nitrides.

\section{METHOD}


The calculations of contacts on 2D materials are carried out using the CASTEP density functional theory (DFT) code [20], using plane waves, pseudopotentials and model periodic supercell models of the systems. We use the Perdew-Burke-Ernzerhof (PBE) form of the generalized gradient approximation (GGA) for the exchange-correlation functional. Normconserving pseudopotentials were used for this purpose with a plane wave cut-off of $490 \mathrm{eV}$. The screened exchange (SX) hybrid functional is used to overcome the band gap errors of the GGA functional [21]. A correction to the GGA treatment of the van der Waals interaction is included by using the Tkatchenko-Scheffler (TS) scheme [22,23] in order to obtain the better bond lengths.

In each case, we created a supercell with a close degree of lattice matching between the nitride layer and the metal contact layers. We used six layers of metal. The vertical size of the supercell includes a vacuum gap of $\mathrm{z}=30 \AA$ (for $\mathrm{X}=\mathrm{B}$ ) or $40 \AA$ (for $\mathrm{X}=\mathrm{Al}, \mathrm{Ga}$ ). The lateral size of the supercell is chosen to attain a reasonable degree of lattice matching between the nitride and the metal layers, while not being so large that it leads to excessive computational costs. Our supercells provide a lattice matching to within 5\%. Table 1 lists the lattice vectors of the metal lattice and the nitride lattice to achieve this matching. The lattice mismatch for each case is given in Tables 2-4. We scale the in-plane lattice constant of the transition metal to match the optimized semiconductor lattice constant.

We then calculate the relaxed atomic structure and then calculated the p-type Schottky barrier height (SBH), which is the energy difference between metal Fermi level and valence band maximum (VBM) of the semiconductor. It can sometimes be difficult to identify the semiconductor VBM in this type of calculation because of the interaction between the semiconductor valence states with the metal states. Sometimes, the semiconductor's residual band structure can be used as a reference marker, as for example in Gong [7]. In other cases, the vacuum level can be used as a reference level to identify a dipole, as in Bokdam [12]. Here, we use a core level of the semiconductor as a reference energy to identify the nitride VBM energy in the interacting system (Fig. 1), the analogue of Kraut's method in photoemission spectroscopy [24]. This method was previously used by us to calculate the Schottky barrier heights for TMDs [11]. The $1 \mathrm{~s}^{2}$ state of boron, $2 \mathrm{~s}^{2}$ of aluminum and $3 \mathrm{~s}^{2}$ of gallium are used for this purpose. Ultra-soft pseudopotentials were generated for this purpose. The plane wave cut-off energy is tested to be $250 \mathrm{eV}, 280 \mathrm{eV}$ and $450 \mathrm{eV}$ for $\mathrm{B}, \mathrm{Al}, \mathrm{Ga}$ respectively. For each system the cut-off energy is chosen to be that necessary to converge the total energy to less than $5 \times 10^{-6} \mathrm{eV} /$ atom. Fig. 2 shows the averaged electrostatic potential for a supercell, which allows us to define the vacuum energy.

\section{RESULTS AND DISCUSSION}

The equilibrium structures of isolated monolayer $\mathrm{h}-\mathrm{XN}$ are relaxed and the calculated lattice parameters are $2.530 \AA$ (h-BN, $1.19 \%$ from experimental result), $3.131 \AA$ (h-AlN) and $3.217 \AA$ (h-GaN).

There are various possible high symmetry bonding geometries between the metal (Me) layers and the nitride that maximize the interaction between these layers, as shown in Fig. 3. Fig. 3(a) shows three possible configurations for large metal atoms like $\mathrm{Sc}$; the metal/ $\mathrm{N} \mathrm{C}_{3 \mathrm{v}}$ symmetry on-top site, the $\mathrm{C}_{\mathrm{s}}$ hollow site, and the $\mathrm{C}_{\mathrm{s}}$ bridge site. For metals like $\mathrm{Ag}$, there are two main sites, Fig 3(b); the $\mathrm{C}_{\mathrm{s}}$ bridge site, and the $\mathrm{C}_{\mathrm{s}}$ on-top/hollow site combination. Other cases have lower symmetry. The energetically most favorable binding configurations are calculated.

The binding energy per molecular unit of different configurations is calculated and these are given in Tables 2-4. Due to the high electronegativity of $\mathrm{N}$ and its preference to bond to the contact metal atoms, the most favorable position for $\mathrm{N}$ and $\mathrm{X}$ are the on-top and hollow sites, respectively. Therefore, in the $1 \times 1$ metal supercells in Fig. 3(a), the $\mathrm{N}$ on-top site is 
favored while for the $\sqrt{3} \times \sqrt{5}$ metal supercells in Fig. 3(b), the on-top and hollow sites are favored. It is always possible to make as many $\mathrm{N}$ atoms on top of metal atoms while keeping the $\mathrm{X}$ away from the metal for other cases in Table 1.

The differences of binding energies between different symmetric configurations are also compared with the thermal perturbation energy. For example, in the Ni|h-AlN and Ni|h-GaN contacts, the planar structures deform most, the N-Ni bonds are shortest and strongest, the largest difference of binding energies between $\mathrm{N}$ on top and other configurations are $10.2 \mathrm{meV} / \AA^{2}$ and $8.2 \mathrm{meV} / \AA^{2}$ respectively. The thermal ripple $\mathrm{kT}$ at room temperature is $\sim 25.6 \mathrm{meV}$ per formula unit, which is larger. Thus it is concluded that the probability of finding each symmetry configuration in experiment is nearly the same for all nitrides.

Fig. 4 shows a side view of the layer contact of $\mathrm{h}-\mathrm{XN}$ with various metals. We show a few cases with the shortest and longest equilibrium distances as examples. The binding energies and layer distances of the most favorable binding sites are given in Tables 2-4.

We now consider the calculated values of Schottky barrier height, as given in Tables 2-4. These are plotted against metal work function in Fig. 5. In order to display three semiconductors SBH on the same diagram, we align their band edges according to their charge neutrality levels (CNLs). The CNL is defined as the branch point energy of the semiconductor where the Greens function of the bulk density of states $N(E)$ is $0[16,25]$ :

$$
\mathrm{G}\left(\mathrm{E}^{n}\right)=\int_{-\infty}^{\infty} \frac{\mathrm{N}\left(\mathrm{E}^{n}\right)}{\mathrm{E}-\mathrm{E}^{i}} \mathrm{~d} \mathrm{E}^{t}=0
$$

The CNL is the energy above which the gap states are empty for a neutral surface. The integral in (1) is formally from $-\infty$ to $\infty$ [17], but in practice we integrate over the valence bands and an equal number of conduction bands [16,25]. Table 5 lists the band gaps and CNLs for the hexagonal XN phases. The resulting band gaps in $\mathrm{SX}$ are $5.76 \mathrm{eV}(\mathrm{h}-\mathrm{BN})$, $3.97 \mathrm{eV}(\mathrm{h}-\mathrm{AlN})$ and $2.02 \mathrm{eV}(\mathrm{h}-\mathrm{GaN})$. Note that these band gaps are smaller than for the $\mathrm{sp}^{3}$ phases. The CNLs lie in the lower half of the gap.

The transition metals used for contacts are the $\mathrm{Sc}, \mathrm{Co}, \mathrm{Ti}(0001)$ surfaces, the $\mathrm{Ag}, \mathrm{Al}, \mathrm{Ni}$, $\mathrm{Pd}, \mathrm{Pt}, \mathrm{Cu}(111)$ surfaces and $\mathrm{MoO}_{3}$. The experimental work function values are taken from Michaelson [26]. The work functions range from $3.5 \mathrm{eV}$ for $\mathrm{Sc}$ to $6.6 \mathrm{eV}$ for $\mathrm{MoO}_{3}$ [26,27]. Despite the different metal reactivities, to be discussed below, the p-type SBH shows a surprisingly good linear relationship against metal work function. The absolute value of slope represents the pinning factor S. Within the MIGS model of pinning, the n-type SBH is given by [18],

$$
\phi_{n}=S\left(\Phi_{M}-\mathrm{E}_{o n t}\right)+E_{o n t}-\chi_{s}
$$

where $\chi_{s}$ is the electron affinity of the semiconductor, $\Phi_{U}$ is the work function of the metal contact, and $E_{\mathrm{cm}}$ is the CNL of the semiconductor. The pinning factor S can vary from 0 (Bardeen limit) to 1 (Schottky limit). $\mathrm{S}=1$ means no pinning in the interface and $\mathrm{S}$ close to 0 means strong pinning. $\mathrm{S}$ can be interpreted in terms of the density of interface gap states which cause pinning, $\mathrm{N}$, as [28]:

$S=\frac{1}{1+\frac{N B_{6}^{2}}{s}}$

where $\delta$ is the decay length of gap states in normal direction and $\varepsilon$ is the local dielectric constant at the interface. A large density of gap states will result in a smaller $\mathrm{S}$ and more pinning. 
The calculated pinning factor of ML h-BN is 0.99 , while that of ML h-AlN is 0.64 and ML h-GaN is 0.63 . This shows that the Fermi level pinning does not occur in h-BN while it is significant in h-AlN and h-GaN. The result of $\mathrm{S}=0.99$ for h-BN is consistent with that of Bokdam et al [12]. However, the dependence of barrier height vs metal work function found here is much more linear, and covers a wider range of work functions.

We now explain how FLP arises in these systems. Pinning requires some charge transfer from the metal to the nitride layer to create a dipole that opposes the change in the work function of the metal. The charge transfer occurs through the overlap of states of the metal and the nitride layer. These states are generally the MIGS. If the separation between the nitride and metal layers is large, the MIGS will have decayed too much at the nitride layer to allow much charge transfer, and the pinning will be small with $\mathrm{S} \sim 1$, Fig 3 . On the other hand, if the layer separation is smaller, there is stronger coupling via chemisorptive bonds between nitride layer and the metal, more charge transfer, and this would give stronger pinning.

Fig 6(a) plots the metal to nitride layer separation against the work function of the metal for all three nitrides. Despite the preference of metal atoms to bond with the nitrogen sites, interestingly there is not much dependence of the separation on the metal work function. Generally, the data points for h-BN stand out from those for h-AlN and h-GaN.

Fig 6(b) plots the binding energy of nitrides to the metal layers against the interlayer separation, $d$. A strong dependence is seen. There is weak binding for $d>2.7 \AA$, but increasingly strong binding for $\mathrm{d}<2.5 \AA$. Clearly, $2.6 \AA$ separates the weakly bound physisorbed layers from the strongly bound chemisorbed layers.

But why do most metals on h-BN fall into the physisorbed category? Fig 6(c) plots the binding energy against the buckling distance of the nitride layer when there is metal on top of it. It is clear that there is only strong binding of the metal layer and short interlayer separation if there is buckling of the nitride layer. The fundamental reason is that h-BN is more stable in its planar $\mathrm{sp}^{2}$ bonded state, with no buckling. In contrast, h-AlN and h-GaN, like their bulk phases are more stable in their $\mathrm{sp}^{3}$ bonded phases, consistent with buckling. The formation of a short, strong bond between the metal and a nitrogen site, making the $\mathrm{N}$ 4-fold coordinated, requires the buckling to occur to facilitate this fourth bond.

We see that there are a few exceptional cases. Ti and Pd are more reactive metals. These are able to buckle h-BN. On the other hand, despite its rather low work function, Ag is a noble metal and it is rather unreactive. It is in the weakly bonded category and causes less buckling on h-AlN or h-GaN than other metals of this work function. A planar $\mathrm{sp}^{2}$ bonded h$\mathrm{XN}$ layer structure is preserved and there is no direct bond formation. Apart from the metal-O bonds in the case of O-rich $\mathrm{MoO}_{3}$, metal-N bonds dominate in Fig. 4.

$\mathrm{MoO}_{3}$ consists of a bilayer of $\mathrm{MoO}$ sites with 3-fold and 2-fold bonded $\mathrm{O}$ sites, 6-fold bonded Mo sites, plus two external layers of monovalent $O$ sites on each side [24]. The interfaces of $\mathrm{MoO}_{3}$ to the nitrides involves contact metal bonds to the external layer oxygen sites of the $\mathrm{MoO}_{3}$ which makes them 2-fold coordinated.

Finally, we can separate the interactions of metals with graphene, nitrides and the TMDs into weak, medium and strongly absorbing, as in Table 6. This uses the data of Popov [3] and Giovannnetti [14] for graphene, and Kang et al [8] and Guo et al [11] for $\mathrm{MoS}_{2}$. We see that the type of interaction is quite similar in each case.

Generally, the Fermi level pinning on the TMDs is stronger than on the nitrides for two reasons. First, the band gaps of the TMDs are smaller, so that the MIGS density of states is larger. Secondly, metal bonding to sulphur atoms of $\mathrm{MoS}_{2}$ occurs directly, it does not require any buckling. Sulfur is able to form extra bonds (overcordinate) being a second row element, whereas nitrogen is a first row element which has less tendency to overcoordinate. 
These three different behaviors occur in all nominally van der Waals bonded 2D systems. Thus, it is not enough to classify them as $2 \mathrm{D}$ or van der Waals solids, it is necessary to consider in greater depth their bonding behaviors.

\section{CONCLUSION}

In summary, the p-type SBHs of the 2D h-XN compounds are calculated using density functional theory. Fermi level pinning is found to occur on defect-free h-AlN and h-GaN layers. The calculated p-type SBH values are consistent with MIGS theory, obeying a linear relationship with the metal work function. Where it occurs, the pinning can be attributed to chemisorptive bonding the metal atoms and the $\mathrm{N}$ sites of the nitrides, which causes a buckling of the planar layers. The Fermi level in h-AlN and h-GaN is pinned near CBM, especially for $\mathrm{h}-\mathrm{GaN}$. The pinning factor for $\mathrm{h}-\mathrm{AlN}$ and $\mathrm{h}-\mathrm{GaN}$ are 0.64 and 0.63 respectively, lower than for h-BN. For h-BN, there is usually no pinning because the highly stable B-N bond opposes buckling, while for h-AlN and h-GaN the pinning is greater due to weaker in-plane bond. It is also found that the N-metal bond as well as equilibrium distance are relevant to FLP. The system with stronger interlayer chemical bond often has stronger FLP.

\section{Acknowledgements}

The authors acknowledge EPSRC and CSC for funding.

\section{REFERENCES}

1. S. Das, A. Prakash, R. Salazar, J. Appenzeller, ACS Nano 8, 1681 (2014).

2. D. Sarkar, X.J. Xie, W. Liu, W. Cao, J.H. Kang, Y.J.Gong, S. Kraemer, P. Ajayan, K. Banerjee, Nature 526, 91 (2015).

3. Y.C. Lin, et al, Nat Commun 6, 7311 (2015)

4. $\quad$ Y Du, L Yang, H Liu, P D Ye, APL Materials 2092510 (2014)

5. S. Das, H. Y. Chen, A. V. Penumatcha, J. Appenzeller, NanoLett 13, 100 (2013)

6. I Popov, G Seifert, D Tomanek, Phys. Rev. Lett. 108, 156802 (2012).

7. C Gong, L Colombo, R M Wallace, K J Cho, Nano Lett, 14: 1714-1720 (2014).

8. J Kang, W Liu, D Sarkar, D Jena, K Banerjee, Phys Rev X 4, 031005 (2014)

9. D. Liu, Y. Guo, L. Fang, J. Robertson App Phys Lett 103183113 (2013)

10. Y Guo, D Liu, J Robertson, App Phys Lett 106173106 (2015)

11. Y Guo, D Liu, J Robertson, ACS Appl Mater Interfaces, 725709 (2015)

12. M Bokdam, G Brocks, M I Katsnelson, P J Kelly, Phys. Rev. B, 90085415 (2014)

13. Y Liu, P Stradins, S H Wei, Sci Adv 2 e1600069 (2016)

14. G Giovannnetti, P A Khomyakov, V M Karpan, J van der Brink, P J Kelly, Phys Rev Lett 101026803 (2008)

15. P A Khomyakov, G Giovannetti, P C Rusu, G Brocks, J van der Brink, P J Kelly, Phys Rev B 79195425 (2009)

16. J Robertson, J Vac Sci Technol B, 181785 (2000).

17. J Tersoff, Phys. Rev. Lett. 52, 465 (1984).

18. W Mönch, Phys. Rev. Lett. 58, 1260 (1987).

19. W Mönch, Surf. Sci. 300, 928-944 (1994).

20. S J Clark, M D Segall, C J Pickard, P J Hasnip, M J Probert, K Refson, M C Payne, Zeitschrift Krist 220, 567 (2005).

21. S J Clark, J Robertson, Phys Rev B 82085208 (2010)

22. A. Tkatchenko and M. Scheffler, Phys. Rev. Lett. 102, 073005 (2009).

23. E R McNellis, J Meyer, K Reuter, Phys. Rev. B 80205414 (2009).

24. E A Kraut, R W Grant, J R Waldrop, Phys. Rev. Letts, 441620 (1980). 
25. J Robertson, B Falabretti, J. Appl. Phys, 1004111 (2006).

26. H B Michaelson, J. Appl. Phys. 48, 4729 (1977).

27. Y Guo, J Robertson, App Phys Lett 105222110 (2014)

28. A W Cowley, S M Sze, J. Appl. Phys 36, 3212 (1965). 
Table 1. In-plane matching of metal and h-XN lattices in each supercell. For example, the Sc $\mid$ h-BN contact $2 \times 2=\sqrt{7} \times \sqrt{7}$ means $2 \times 2$ double size supercell of Scandium (0001) surface is fitted into a $\sqrt{7} \times \sqrt{7}$ supercell of h-BN.

\begin{tabular}{llll} 
Transition metal & $h-B N$ & $h-A I N$ & $h-G a N$ \\
\hline Sc(0001) & $2 \times 2=\sqrt{7} \times \sqrt{7}$ & $1 \times 1=1 \times 1$ & $1 \times 1=1 \times 1$ \\
$A g(111)$ & $\sqrt{3} \times \sqrt{3}=2 \times 2$ & $2 \times 2=\sqrt{3} \times \sqrt{3}$ & $2 \times 2=\sqrt{3} \times \sqrt{3}$ \\
Al(111) & $\sqrt{3} \times \sqrt{3}=2 \times 2$ & $2 \times 2=\sqrt{3} \times \sqrt{3}$ & $2 \times 2=\sqrt{3} \times \sqrt{3}$ \\
$\operatorname{Ti}(0001)$ & $\sqrt{3} \times \sqrt{3}=2 \times 2$ & - & - \\
$\mathrm{Cu}(111)$ & $1 \times 1=1 \times 1$ & $2 \times 2=\sqrt{3} \times \sqrt{3}$ & $\sqrt{7} \times \sqrt{7}=2 \times 2$ \\
$\mathrm{Co}(0001)$ & $1 \times 1=1 \times 1$ & $\sqrt{7} \times \sqrt{7}=2 \times 2$ & $\sqrt{7} \times \sqrt{7}=2 \times 2$ \\
$\mathrm{Pd}(111)$ & $\sqrt{3} \times \sqrt{3}=2 \times 2$ & - & - \\
$\mathrm{Ni}(111)$ & $1 \times 1=1 \times 1$ & $\sqrt{7} \times \sqrt{7}=2 \times 2$ & $\sqrt{7} \times \sqrt{7}=2 \times 2$ \\
$\mathrm{Pt}(111)$ & $\sqrt{7} \times \sqrt{7}=3 \times 3$ & $2 \times 2=\sqrt{3} \times \sqrt{3}$ & $2 \times 2=\sqrt{3} \times \sqrt{3}$ \\
$\mathrm{MoO}_{3}$ & $4 \times 6=3 \times 5 \sqrt{3}$ & $4 \times 3=5 \times 2 \sqrt{3}$ & $5 \times 3=6 \times 2 \sqrt{3}$ \\
\hline
\end{tabular}

Table 2. Comparison of band gaps calculated by PBE and sX-LDA and CNLs extracted from sX bands

\begin{tabular}{llll} 
Semiconductor & \multicolumn{2}{l}{ Band gap (eV) } & Isotropic \\
\cline { 2 - 4 } & PBE & sX-LDA & CNL(eV), sX \\
\cline { 2 - 3 } ML h-BN & 4.77 & 5.72 & 2.12 \\
ML h-AIN & 2.92 & 3.84 & 2.18 \\
ML h-GaN & 1.88 & 3.19 & 1.33 \\
\hline
\end{tabular}

Table 3. Details of metal h-BN contact. The lattice mismatch is the difference between the lengths of two supercells divided by length of the bigger supercell. The binding energy is the energy per formula unit for binding the two materials together. The equilibrium distance is the normal plane - top plane separation between h-BN layer and the surface of metals.

\begin{tabular}{|c|c|c|c|c|c|}
\hline Transition metal & $\begin{array}{l}\text { Work } \\
\text { function (eV) }\end{array}$ & $\begin{array}{l}\text { Lattice } \\
\text { mismatch (\%) }\end{array}$ & $\begin{array}{l}\text { Binding } \\
\text { energy } \\
(e V / X N)\end{array}$ & $\begin{array}{l}\text { Layer } \\
\text { distance (Å) }\end{array}$ & $\begin{array}{l}\text { P-type } \\
\text { SBH(eV) }\end{array}$ \\
\hline$S c(0001)$ & 3.50 & 1.17 & -0.19 & 3.57 & 3.43 \\
\hline$A g(111)$ & 4.26 & 1.11 & -0.85 & 3.05 & 2.74 \\
\hline$A l(111)$ & 4.28 & 1.99 & -0.22 & 3.22 & 2.59 \\
\hline Ti(0001) & 4.33 & 0.99 & -0.53 & 2.01 & 2.46 \\
\hline$C u(111)$ & 4.65 & 1.01 & -0.19 & 2.99 & 2.13 \\
\hline $\mathrm{Co}(0001)$ & 5.00 & 0.91 & -0.39 & 3.05 & 1.78 \\
\hline$P d(111)$ & 5.12 & 5.83 & -0.24 & 2.36 & 1.83 \\
\hline $\mathrm{Ni}(111)$ & 5.15 & 1.51 & -0.27 & 3.02 & 1.71 \\
\hline $\operatorname{Pt}(111)$ & 5.65 & 3.29 & -0.01 & 3.51 & 1.51 \\
\hline $\mathrm{MoO}_{3}$ & 6.61 & $\begin{array}{l}4.23(x) \\
1.20(y)\end{array}$ & -0.22 & 2.85 & -0.03 \\
\hline
\end{tabular}


Table 4. List of details of h-AlN metal contact.

\begin{tabular}{|c|c|c|c|c|c|}
\hline Transition metal & $\begin{array}{l}\text { Work } \\
\text { function (eV) }\end{array}$ & $\begin{array}{l}\text { Lattice } \\
\text { mismatch (\%) }\end{array}$ & $\begin{array}{l}\text { Binding } \\
\text { energy } \\
(\mathrm{eV} / \mathrm{XN})\end{array}$ & $\begin{array}{l}\text { Layer } \\
\text { distance (A) }\end{array}$ & $\begin{array}{l}\text { P-type } \\
\text { SBH(eV) }\end{array}$ \\
\hline$S c(0001)$ & 3.50 & 5.3 & -1.22 & 2.30 & 2.15 \\
\hline$A g(111)$ & 4.26 & 4.4 & -0.89 & 2.70 & 1.68 \\
\hline$A l(111)$ & 4.28 & 5.3 & -0.73 & 2.04 & 2.30 \\
\hline $\mathrm{Cu}(111)$ & 4.65 & 5.7 & -0.74 & 2.22 & 1.65 \\
\hline $\mathrm{Co}(0001)$ & 5.00 & 5.6 & -1.14 & 2.05 & 1.23 \\
\hline $\mathrm{Ni}(111)$ & 5.15 & 5.0 & -1.14 & 2.04 & 1.32 \\
\hline $\operatorname{Pt}(111)$ & 5.65 & 2.3 & -1.02 & 2.24 & 1.00 \\
\hline $\mathrm{MoO}_{3}$ & 6.61 & $\begin{array}{l}1.23(x) \\
2.05(v)\end{array}$ & -0.35 & 1.90 & 0.28 \\
\hline
\end{tabular}

Table 5. List of details of h-GaN metal contact.

\begin{tabular}{|c|c|c|c|c|c|}
\hline Transition metal & $\begin{array}{l}\text { Work } \\
\text { function (eV) }\end{array}$ & $\begin{array}{l}\text { Lattice } \\
\text { mismatch (\%) }\end{array}$ & $\begin{array}{l}\text { Binding } \\
\text { energy } \\
(\mathrm{eV} / \mathrm{XN})\end{array}$ & $\begin{array}{l}\text { Layer } \\
\text { distance (A) }\end{array}$ & $\begin{array}{l}\text { P-type } \\
\text { SBH(eV) }\end{array}$ \\
\hline$S c(0001)$ & 3.50 & 2.8 & -1.34 & 2.21 & 1.87 \\
\hline$A g(111)$ & 4.26 & 3.6 & -0.64 & 2.67 & 1.65 \\
\hline$A l(111)$ & 4.28 & 2.7 & -0.67 & 1.99 & 1.84 \\
\hline $\mathrm{Cu}(111)$ & 4.65 & 4.9 & -0.70 & 2.18 & 1.31 \\
\hline $\mathrm{Co}(0001)$ & 5.00 & 3.0 & -1.19 & 2.04 & 0.99 \\
\hline $\mathrm{Ni}(111)$ & 5.15 & 2.4 & -1.63 & 2.03 & 1.35 \\
\hline $\operatorname{Pt}(111)$ & 5.65 & 0.4 & -0.95 & 2.17 & 0.67 \\
\hline $\mathrm{MoO}_{3}$ & 6.61 & $\begin{array}{l}2.60(x) \\
0.48(y)\end{array}$ & -0.31 & 2.20 & 0.04 \\
\hline
\end{tabular}

Table 6. Three classes of adsorption types.

\begin{tabular}{|c|c|c|c|c|c|c|}
\hline \multirow{2}{*}{$\begin{array}{l}\text { Semiconductor } \\
\text { monolayer } \\
\text { Contact situation }\end{array}$} & \multirow{2}{*}{$\begin{array}{l}\text { Graphene }^{\mathbf{2 1}} \\
\text { PBE }\end{array}$} & \multicolumn{2}{|l|}{$T_{M D}^{22}$} & \multicolumn{3}{|l|}{ h-XN } \\
\hline & & MoS2 & WSe2 & $\mathrm{BN}$ & AIN & GaN \\
\hline Weak & $\begin{array}{l}\mathrm{Al}, \mathrm{Cu}, \mathrm{Ag}, \mathrm{Au}, \\
\mathrm{Pt}\end{array}$ & In, Au & In, Au & $\begin{array}{l}\mathrm{Sc}, \mathrm{Cu}, \mathrm{Ag}, \mathrm{Al}, \\
\mathrm{Co}, \mathrm{Pt}, \mathrm{Ni}, \\
\mathrm{MoO}_{3}\end{array}$ & & \\
\hline Medium & --- & $\mathrm{Pd}$ & $\mathrm{Ti}, \mathrm{Pd}$ & & $\mathrm{Ag}$ & $\mathrm{Ag}$ \\
\hline Strong & $\mathrm{Co}, \mathrm{Ni}, \mathrm{Pd}$ & $\begin{array}{l}\text { Ti, Mo, } \\
\text { W }\end{array}$ & Mo, W & $\mathrm{Ti}, \mathrm{Pd}$ & $\begin{array}{l}\mathrm{Sc}, \mathrm{Cu}, \mathrm{Pt}, \\
\mathrm{Al}, \mathrm{Co}, \mathrm{Ni}, \\
\mathrm{MoO}_{3}\end{array}$ & $\begin{array}{l}\mathrm{Sc}, \mathrm{Cu}, \mathrm{Pt}, \\
\mathrm{Al}, \mathrm{Co}, \mathrm{Ni} \text {, } \\
\mathrm{MoO}_{3}\end{array}$ \\
\hline
\end{tabular}


Figure captions

1. Schematic of core level reference method. Energy bands are shown as colored blocks.

2. Averaged electronic potential of h-BN, h-AlN and h-GaN monolayers adsorbed on six layers of $\mathrm{Cu}$ (111) surface. The green line is vacuum level and the red line is the Fermi level. The potential level coincides with the vacuum level in vacuum region, which shows the vacuum is thick enough. Inside the layers, the potential oscillates with the atom layers. Note the longer decay length of metal induced gap states between the metal and h-BN layer than for the other nitrides.

3. Top view of possible top contact binding configurations with symmetries. Only the top layer of metals is shown. (a) is the $1 \times 1 \mathrm{~h}-\mathrm{GaN}$ matching with $1 \times 1 \mathrm{Sc}(0001)$ surface,

(b) is the $2 \times 2$ h-BN matching with $\sqrt{3} \times \sqrt{3} \mathrm{Ag}$ (111) surface. The color of atom, $\mathrm{B}=$ peach, $\mathrm{N}=$ blue, $\mathrm{Sc}=$ light gray, $\mathrm{Ga}=$ brown, $\mathrm{Ag}=$ light blue. (c) The $1 \times 1$ cell lattice $\alpha$ of metal and $\mathrm{GaN}$ in (a), and metal in (b) is marked red, while the $2 \times 2 \mathrm{BN}$ lattice or the $\sqrt{3} \times \sqrt{3}$ Ag lattice $\beta$ in (b) is marked green.

4. Top contact bonding at (a)/(b) Ti/Sc-BN interface, (c)/(d) $\mathrm{MoO}_{3} / \mathrm{Ag}-\mathrm{AlN}$ interface and (e)/(f) $\mathrm{Al} / \mathrm{Ag}-\mathrm{GaN}$ interface. The color of atom, $\mathrm{B}=$ peach, $\mathrm{N}=$ blue, $\mathrm{O}=\mathrm{red}, \mathrm{Al}=$ fuchsia pink, $\mathrm{Sc}=$ light grey, $\mathrm{Ti}=$ dark gray, $\mathrm{Mo}=$ cyan, $\mathrm{Ga}=$ brown, $\mathrm{Ag}=$ light blue. The shortest distance is marked as a red line. The upper half is classified as chemisorption while the lower half is physisorption, with no bond. Distances in Angstroms.

5. P-type SBH of metal-h-XN contact, referred to each XN's CNLs, colors of data points and their band edges, with an ideal linear fitting with pinning factor of $0.99(X=B), 0.64$ $(\mathrm{X}=\mathrm{Al}), 0.63(\mathrm{X}=\mathrm{Ga})$ and standard error of $0.06,0.09,0.08$, respectively.

6. (a) Metal work function (W) vs equilibrium distances between $\mathrm{XN}$ and metal layers (d).

(b) $\mathrm{d}$ vs Buckling. (c) $\mathrm{d}$ vs binding energies. Metal and $\mathrm{N}$ form bond when $\mathrm{XN}$ is chemisorbed onto metals, except the case of $\mathrm{MoO}_{3} \mathrm{O}$-rich, where metal and oxygen tend to form bonds. The grey region shows a transition from physisorption to chemisorption. For $\mathrm{X}=\mathrm{B}$, the distances are mainly above the grey region. Ti and $\mathrm{Pd}$ are two exceptions, marked as red in (a). For $\mathrm{X}=\mathrm{Al}$ and $\mathrm{Ga}$, the distances are mainly below the grey region. Exceptions are Ag which is marked as blue in (a). 


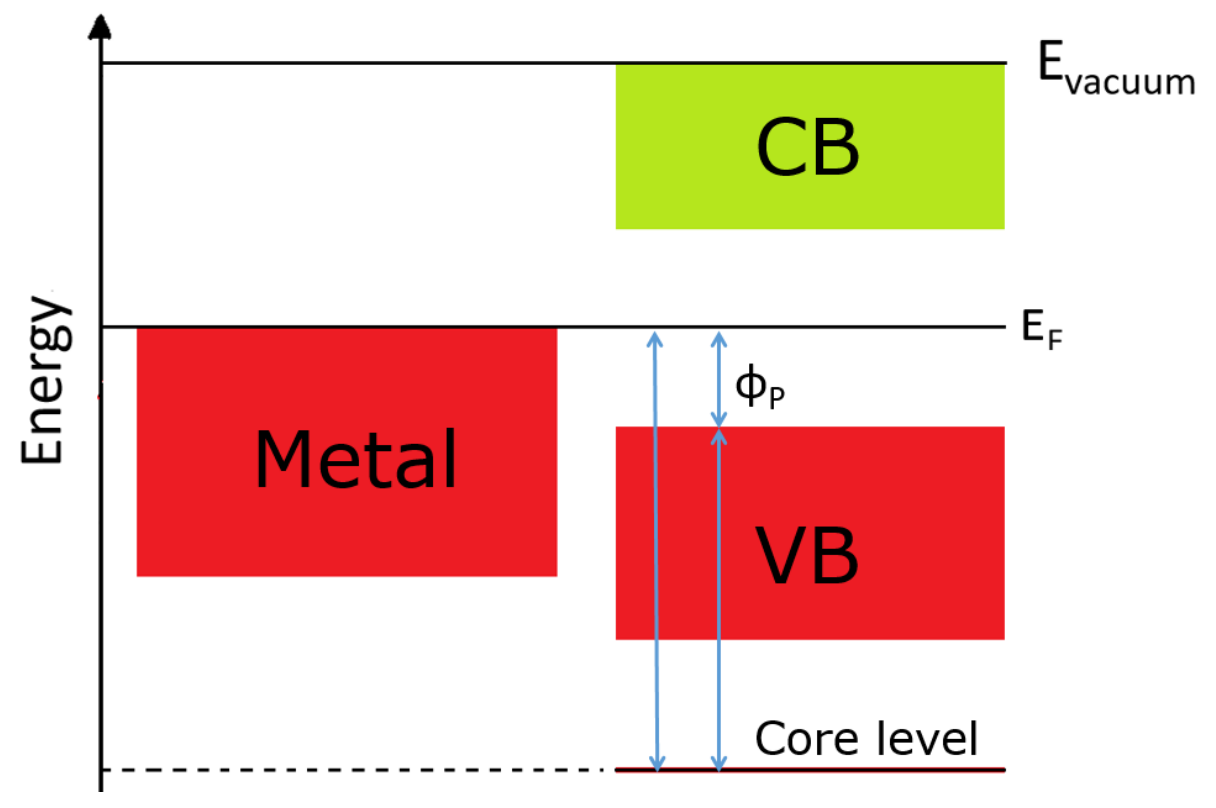

Figure 1 

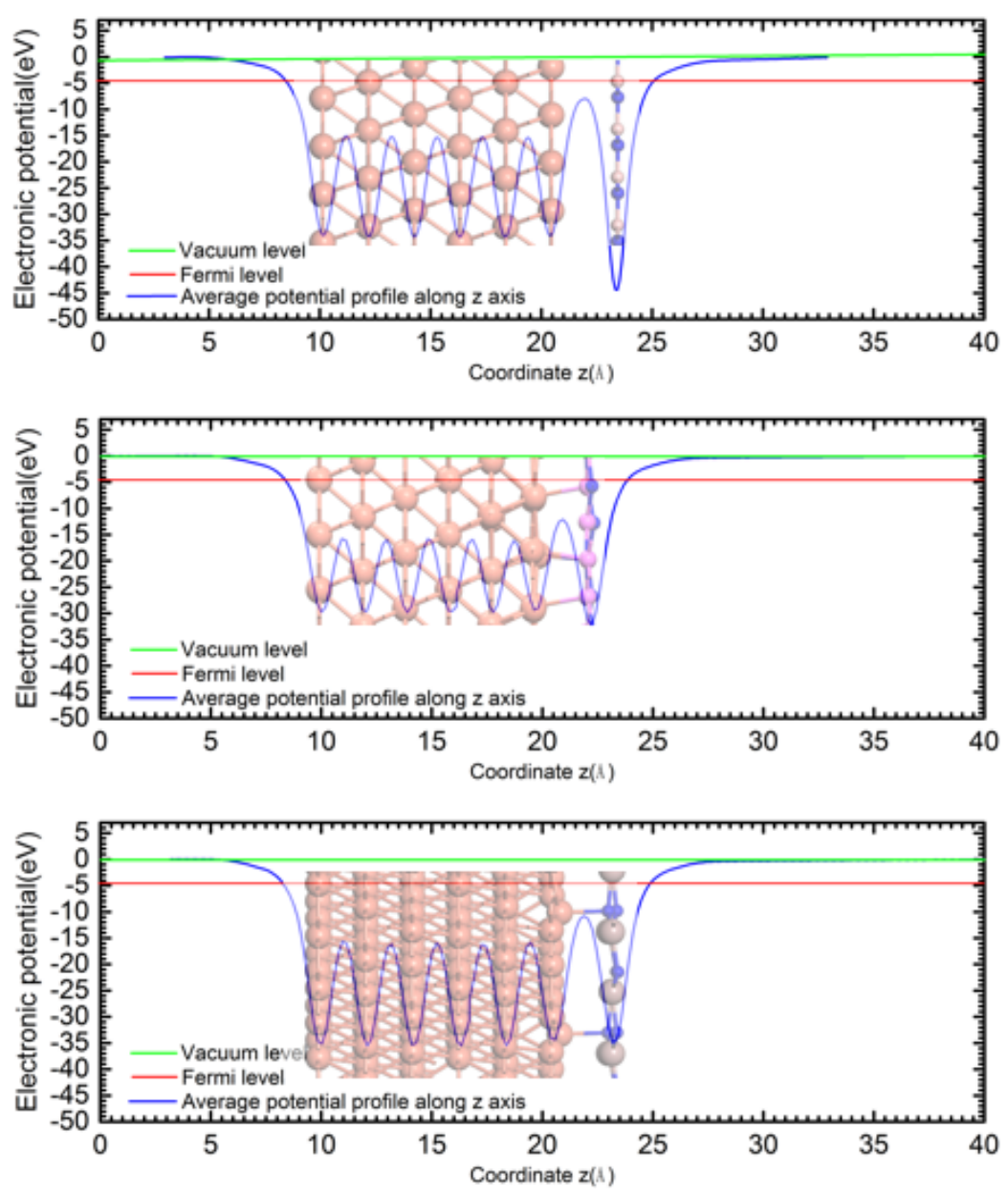

Figure 2 
High symmetry absorption geometries

(a)

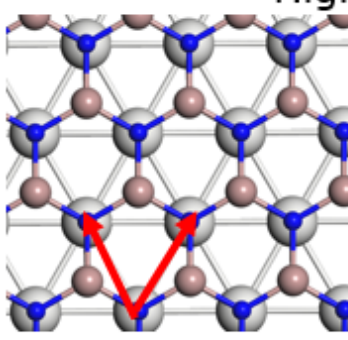

On top

(b)

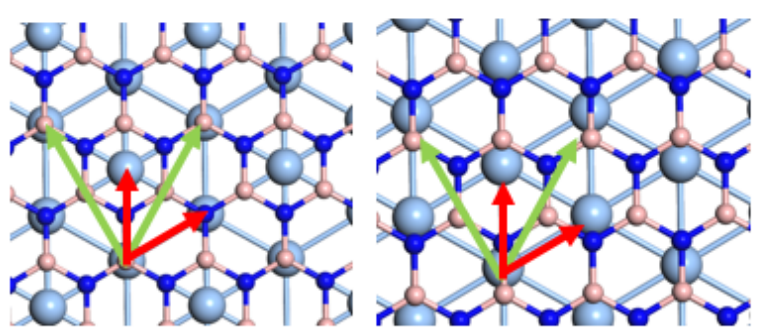

Bridge sites On top and Hollow sites

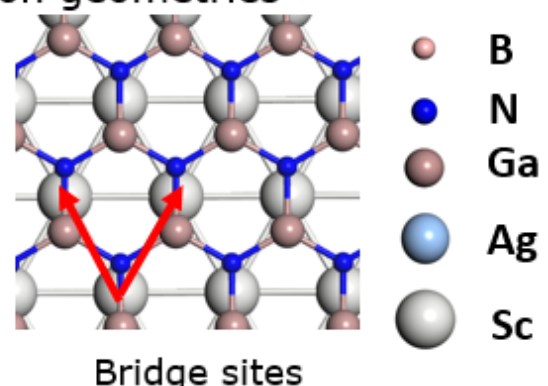

(c)

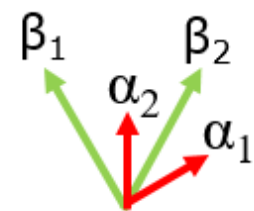

Cell lattice

Figure 3 


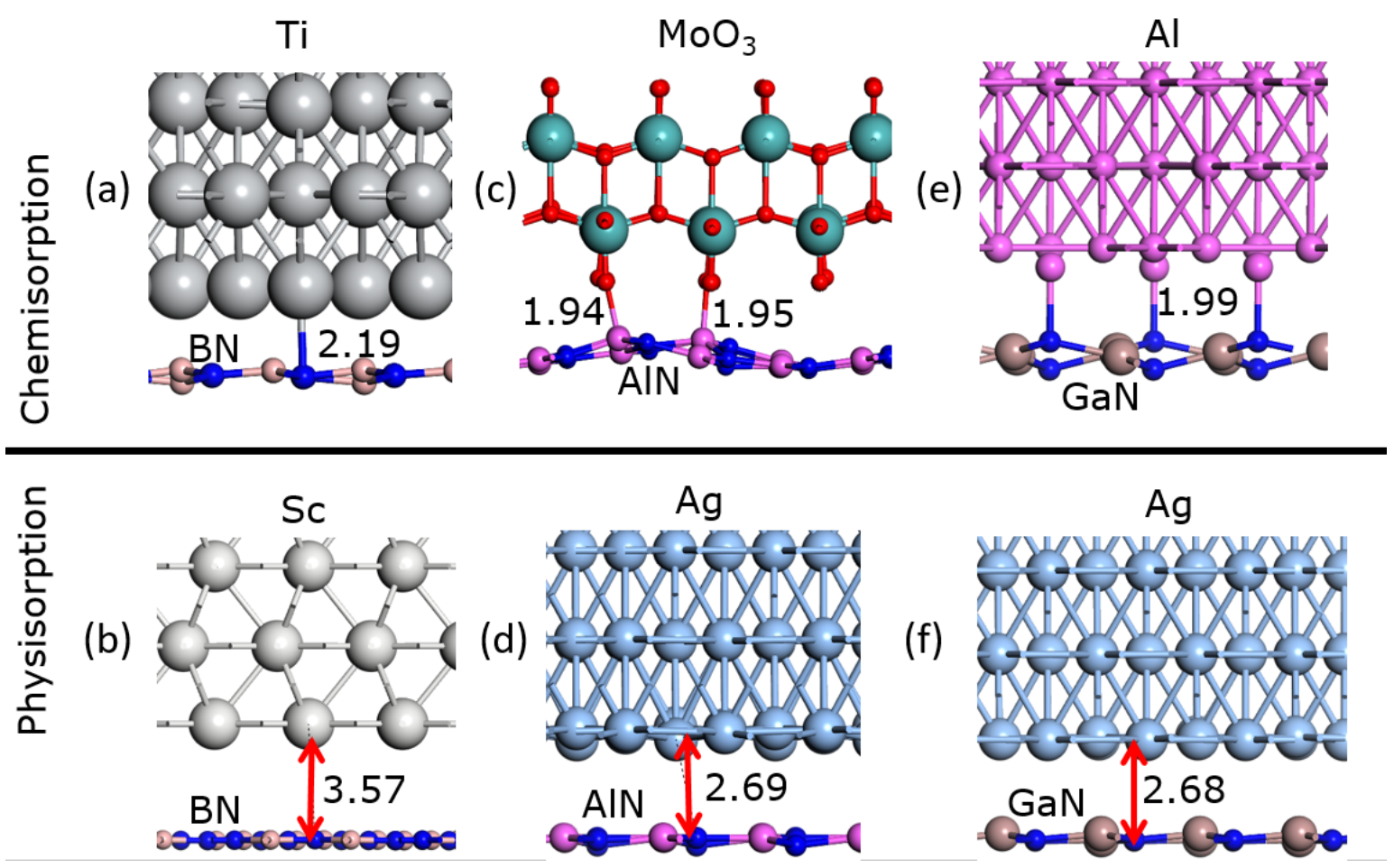

Figure 4 


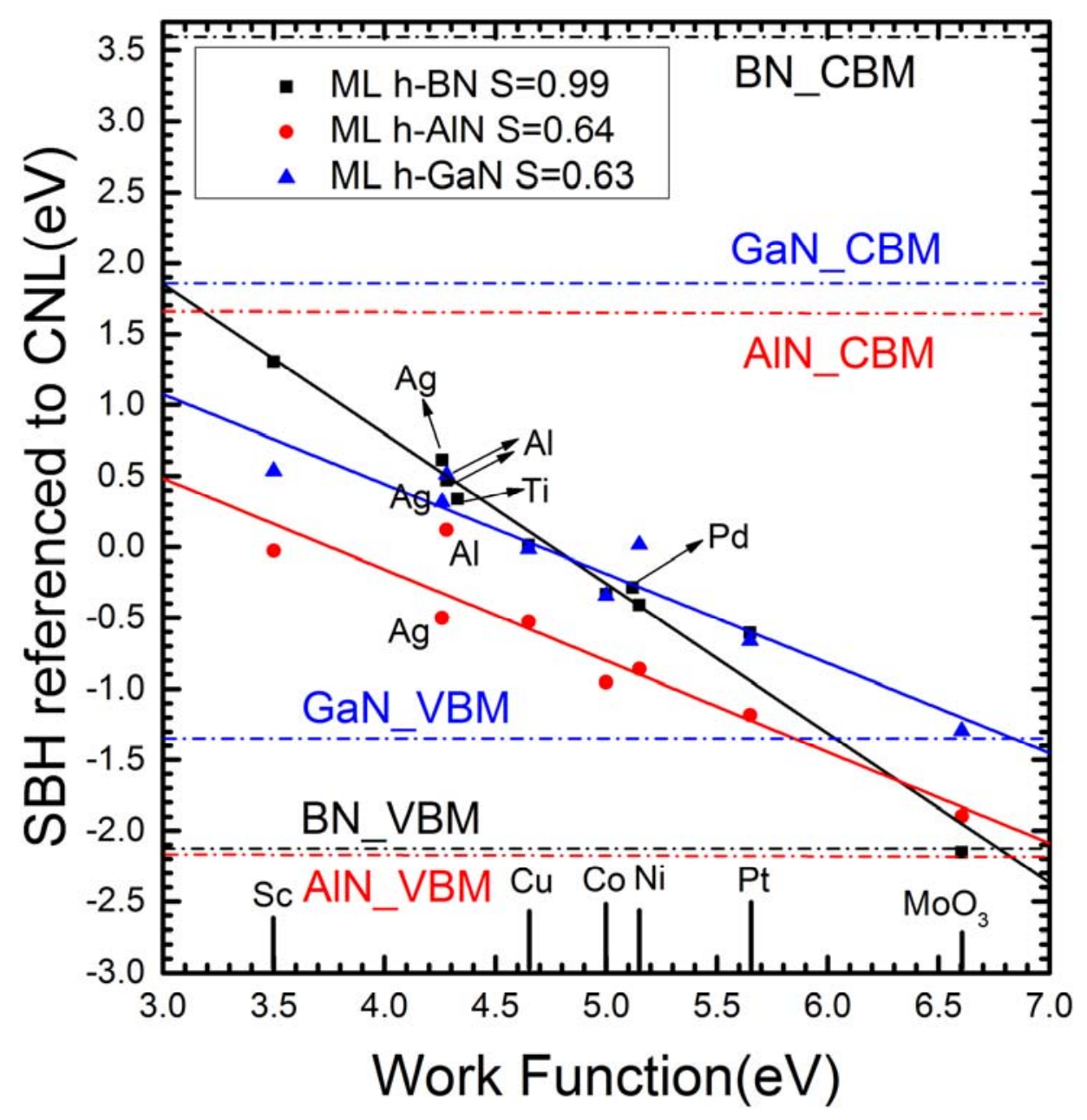

Figure 5 


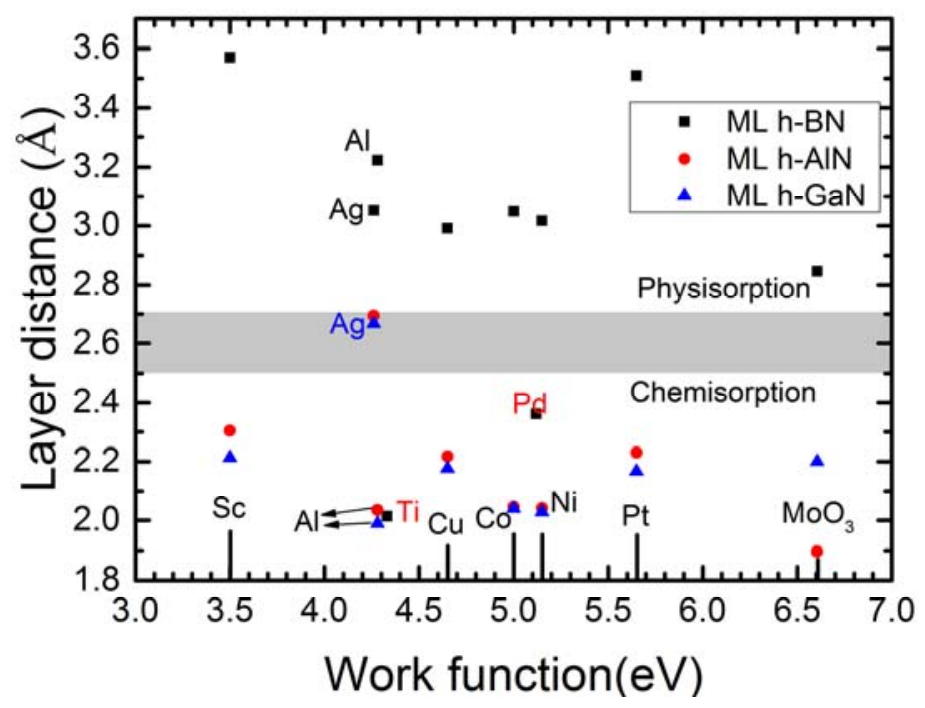

(a)

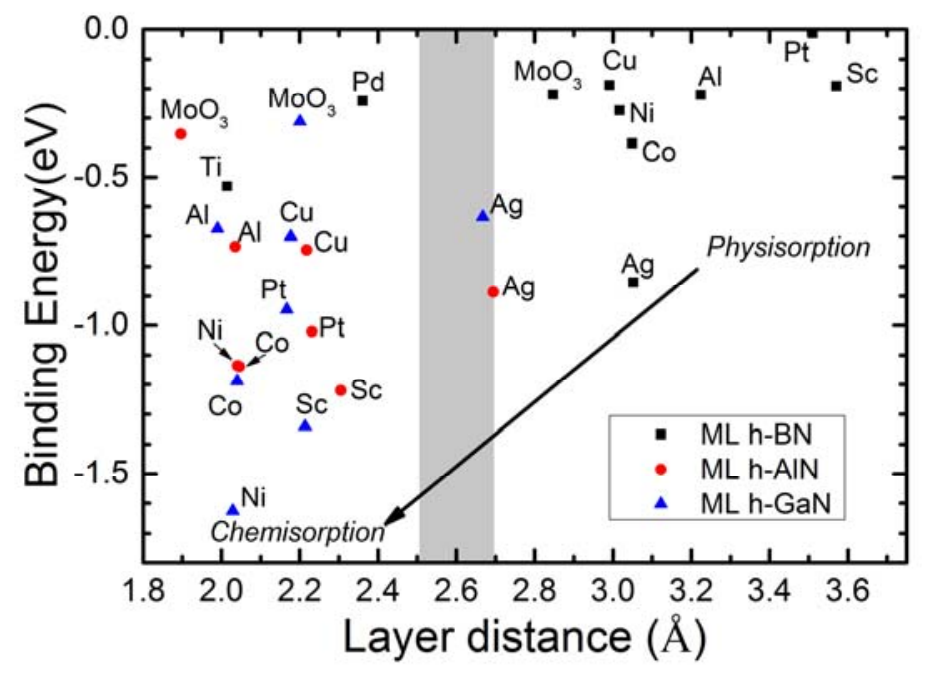

(b)

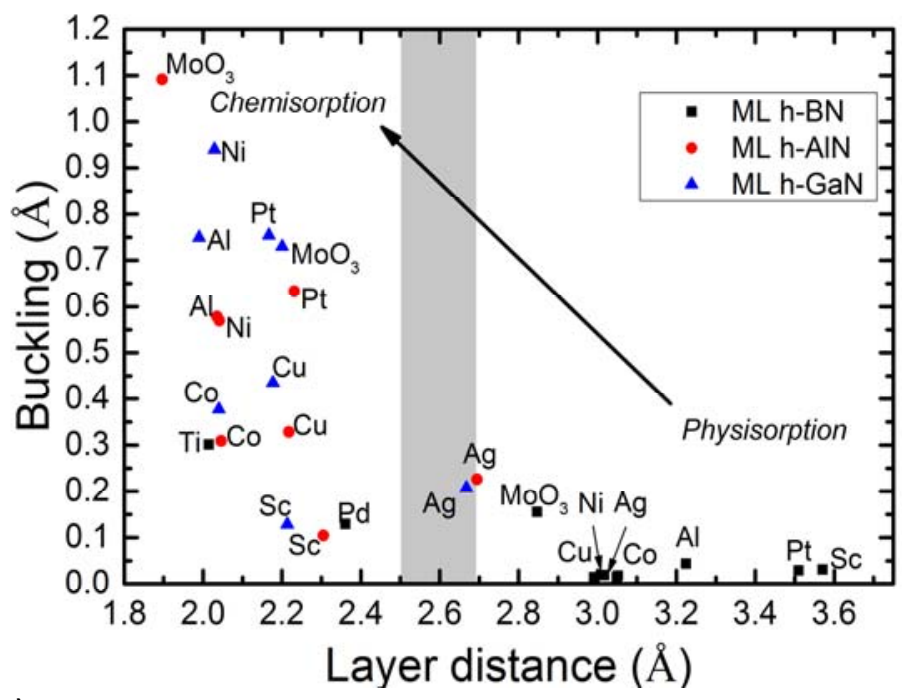

(c)

Figure 6 